\title{
EXPEDIENTE
}

\section{EDITOR CIENTÍFICO}

Celia Linhares, UFRRJ/PPGEDUC, Brasil

\section{COMISSÃO EDITORIAL}

Carmen Sanches, Universidade Federal do Estado do Rio de Janeiro (UNIRIO), Brasil Denize Sepúlveda, FFP/UERJ, Brasil

Flavia Miller Naethe Motta, UFRRJ - PPGEDUC, Brasil

Lucia Velloso, FFP/UERJ, Brasil

Marcia Pletsch, UFRRJ/IM

Rosimeri de Oliveira Dias, FFP/UERJ, Brasil

\section{EDITORES GERENTES}

Flavia Miller Naethe Motta, UFRRJ - PPGEDUC, Brasil

Rosimeri de Oliveira Dias, FFP/UERJ, Brasil

Carmen Sanches, Universidade Federal do Estado do Rio de Janeiro (UNIRIO), Brasil

EDITORES DOSSIÊ 50 ANOS DE PEDAGOGIA DO OPRIMIDO: MOVIMENTOS DE OPRESSÕES E EMANCIPAÇÕES CONTEMPORÂNEAS NA AMÉRICA LATINA E ÁFRICA

Márcia Soares de Alvarenga, FFP/UERJ, Brasil

Maria Tereza Goudard Tavares, FFP/UERJ, Brasil

\section{CONSELHO EDITORIAL}

Prof $^{a} \operatorname{Dr}^{\mathrm{a}}$ Ana Maria Monteiro, UFRJ, Brasil

Prof. Dr. Carlos Eduardo Ferraço, Professor Associado da UFES, Brasil

Prof. Dr. Carlos Skliar, FLACSO-Argentina, Brasil

Prof $^{a}$ Dr $^{\mathrm{a}}$ Catherine Walsh, Profesora de la Universidad Andina Simón Bolivar/Equador, Equador

Prof $^{a}$ Dr $^{\mathrm{a}}$ Filomena Maria de Arruda Monteiro, Professora Associada da UFMT, Brasil

Prof. Dr. Guilherme do Val Toledo Prado, UNICAMP, Brasil

Prof $^{a}$ Dr $^{\mathrm{a}}$ Inês Assunção de Castro Teixeira, Professora Associada da UFMG, Brasil

Prof $^{a} \operatorname{Dr}^{\mathrm{a}}$ Maura Corcini Lopes

Prof $^{a}$ Dr $^{\mathrm{a}}$ Maria Teresa Esteban, Professora Associada da UFF, Brasil

Prof. Dr. Marcos Reigotta, Professor Titular da UNISO, Brasil

Prof Nelson De Luca Pretto, Professor Titular da Faculdade de Educação da Universidade

Federal da Bahia, Brasil

Prof $^{a} \operatorname{Dr}^{\mathrm{a}}$ Nilma Lino Gomes, Professora Adjunta da UFMG, Brasil

Prof $^{a}$ Dr $^{a}$ Virginia de Oliveira Silva, Professora Adjunto da UFPB, Brasil 
AVALIADORES AD HOC DO DOSSIÊ

Adriana Almeida

Amanda Motta Castro

Ana Patrícia Silva

Ana Paula Moura

Ana Paula Abreu Moura

Arthur Vianna Ferreira

Beatriz Sancovschi

Carmen Lúcia Vidal Pérez

Clarissa Craveiro

Denise Rezende

Edna Oliveira

Eliane Fernandes

Fabiana Eckhardt

Flávio Anício Andrade

Glicilene Barão

Guilherme do Val Toledo Prado

Jaqueline Ventura

José Antonio Miranda Sepulveda

Márcia Maria Silva

Rafael Marques Gonçalves

Rafael Mendes

Rosa Malena Carvalho

Rosa Pinheiro

Sandra Almeida

Vania Finholdt Leite 